\title{
Resultados a largo plazo del tratamiento con quimioterapia y radioterapia en linfoma no Hodgkin localizado, de grados intermedio y alto
}

\author{
Pablo Ramírez $\mathbf{V}^{1}$, Mauricio 0 cqueteau $\mathrm{T}^{1}$, \\ Manuel Alvarez $\mathrm{Z}^{1}$, Pablo Bertín C-M ${ }^{1}$, Pablo Lira $\mathbf{V}^{1}$, \\ Marisa Bustos $C^{2}$, Pelayo Besa de $C^{2}$. \\ Long term results for intermediate \\ and high grade localized non \\ Hodgkin lymphoma, treated with \\ chemotherapy and radiotherapy
}

Background: Treatment of intermediate and high grade nonHodgkin lymphoma (NHL) includes chemotherapy with or without radiotherapy, depending on the clinical stage. The standard treatment for advanced NHL is 8 cycles of combined chemotherapy, cyclophosphamide, adriamicin, vincristine and prednisone (CHOP). Patients presenting with localized disease are treated with fewer chemotherapy cycles and involved field radiotherapy, with good results. Aim: To evaluate the treatment results including overall survival (OS) and event-free survival (EFS) in localized aggressive NHL patients treated at the Pontificia Universidad Católica de Chile, Clinical Hospital. Patients and Methods: Retrospective analysis of all patients with Ann Arbor stages I and II referred to the hematology and radiotherapy clinic between 1998 and 2003. OS and EFS analysis was made according to the Kaplan and Meier method. Log-rank and Cox methods were used for univariate and multivariate analyses, respectively. Chemotherapy and radiotherapy toxicities were scored according to World Health Organization (WHO) and Radiation Therapy Oncology Group (RTOG) scales, respectively. Results: 39 patients ( 20 men), aged between 20 to 85 years, were the source for this study. The average follow-up was 51 months (range 6-115). The 5 years OS and EFS were $72,4 \%$ and $63,3 \%$, respectively. On univariate analysis, age over 60 was the only variable that affected negatively OS and EFS. Acute toxicity caused by chemotherapy and radiotherapy was uncommon. Conclusions: Age over 60 was the only independent variable associated with poor prognosis. The number of chemotherapy cycles and the drug combination did not influence the results. These results support the usefullness of a shortened chemotherapy regimen plus involved field radiotherapy (Rev Méd Chile 2006; 134: 1409-16).

(Key words: Chemotherapy; Lymphoma, non-Hodgkin; Radiotherapy)

Recibido el 29 de noviembre, 2005. Aceptado el 21 de abril, 2006.

${ }^{1}$ Departamento de Hematología Oncología, ${ }^{2}$ Departamento de Radiología, Servicio de Radio-Oncología, Centro de Cáncer, Facultad de Medicina, Pontificia Universidad Católica de Chile. Santiago de Chile.

Correspondencia a: Dr. Pelayo Besa de Cárcer. Diagonal Paraguay 319, Centro de Cáncer, Departamento de Radiología, Servicio de Radio-Oncología, Pontificia Universidad Católica de Chile. Teléfono: 354 6841. Fax: 247 2327. E mail: pbesa@med.puc.cl 
L os linfomas no Hodgkin (LNH) agresivos son un grupo de tumores malignos potencialmente curables con poliquimioterapia en base a doxorrubicina ${ }^{1}$. Los factores pronóstico más relevantes están incluidos en el Indice Pronóstico Internacional (IPI) y son: la edad del paciente, la capacidad funcional, la etapa clínica según la clasificación de Ann Arbor, y compromiso tumoral extralinfático ${ }^{2}$. Dentro de éstos el más importante es la de una masa tumoral superior a $10 \mathrm{~cm}$ de diámetro mayor. Diversos estudios han demostrado que el tratamiento de pacientes en etapas Ann Arbor I y II, nodales y extranodales, tienen resultados superiores en sobrevida que aquellos pacientes similares pero con enfermedad tumoral mayor a $10 \mathrm{~cm}$, por lo que en la actualidad se definen como etapas localizadas las etapas Ann Arbor I y II inferiores a $10 \mathrm{~cm}$, y como enfermedad avanzada a las etapas III, IV y las etapas I y II mayores a $10 \mathrm{~cm}^{3,4}$.

Antes de 1970 el tratamiento de los LNH agresivos en etapas localizadas era radioterapia ${ }^{5}$. Los resultados eran insatisfactorios, con sobrevida global (SG) a 5 años no superior a $40 \%$ para la etapa $\mathrm{II}^{6}$. A fines de los 70 se demostró que para el subgrupo de LNH difusos los esquemas de quimioterapia en base a doxorrubicina eran superiores en el porcentaje de respuestas completas y sobrevida global que los sin doxorrubicina ${ }^{7}$. En la década de los 80 , buscando disminuir la toxicidad de los tratamientos sin disminuir el porcentaje de curación, distintos autores demostraron que en pacientes portadores de LNH de grado intermedio y alto, clínicamente localizados, tratados con quimioterapia CHOP (ciclofosfamida, doxorrubicina, vincristina, prednisona) con o sin radioterapia a los sitios comprometidos, podían evitar la laparotomía exploradora de etapificación, obteniéndose resultados superiores a los observados con radioterapia exclusiva, y con menor morbili$\mathrm{dad}^{8-10}$

En 1993 mediante un estudio multicéntrico se demostró que el esquema CHOP era igualmente efectivo en cuanto a sobrevida libre de eventos (SLE) y sobrevida global que los esquemas llamados de segunda y tercera generación, pero con una morbilidad asociada significativamente menor, estableciéndose a esta combinación como el estándar de tratamiento en LNH avanzados ${ }^{1}$. Posteriormente este esquema también se siguió utilizando en el tratamiento de LNH localizados.
Es sólo a fines de los 90, en que en la búsqueda para disminuir la toxicidad de la quimioterapia, Miller demostró que para etapas localizadas, 3 ciclos de quimioterapia $\mathrm{CHOP}$ seguido de radioterapia a las zonas comprometidas eran más efectivos que 8 ciclos de quimioterapia CHOP, y con una menor toxicidad ${ }^{11}$. Respecto del uso de otros esquemas de quimioterapia en LNH localizados no existe información en base a estudios randomizados por lo que no existe comparación entre los esquemas CHOP y EPOCH (etopósido, prednisona, vincristina, ciclofosfamida, doxorrubicina) acortados asociados a radioterapia a los sitios comprometidos por tumor, siendo el segundo esquema una alternativa de tratamiento que se ha utilizado en nuestro grupo.

En este estudio presentamos los resultados retrospectivos de la evolución de pacientes portadores de LNH localizados, agresivos, menores a 10 $\mathrm{cm}$ de diámetro mayor, tratados en nuestra institución entre los años 1996 y 2003 con quimioterapia en base a doxorrubicina y radioterapia a los sitios comprometidos por tumor. El objetivo primario es analizar la SLE y la SG, y determinar si existen diferencias entre los distintos esquemas de tratamiento, y la influencia de distintos factores pronósticos. Como objetivos secundarios se describen las toxicidades agudas de la quimioterapia $\mathrm{y}$ radioterapia.

\section{PACIENTES Y MÉTODOS}

Se analizaron retrospectivamente los registros de todos los pacientes mayores de 18 años, portadores de LNH estirpe $B$, en etapas I y II según la clasificación de Ann Arbor ${ }^{12}$, de grados intermedio y alto según la clasificación de la OMS (Organización Mundial de la Salud) ${ }^{13}$, cuya biopsia fue revisada en nuestro servicio de anatomía patológica, y que hayan recibido tratamiento en el Centro de Cáncer de la Pontificia Universidad Católica, con quimioterapia CHOP o EPOCH, por al menos 3 ciclos, y radioterapia a los campos comprometidos. Para la radioterapia se utilizaron rayos X de 6 ó $20 \mathrm{MV}$, a los sitios comprometidos, entregando una dosis de 39.6 Gy en 22 fracciones (Tabla 1). Se excluyeron los pacientes con enfermedad superior a $10 \mathrm{~cm}$ de diámetro mayor por corresponder a la etapa avanzada. 
Tabla 1. Esquemas de tratamiento administrados a pacientes con LN H localizado de intermedio y alto grado

\begin{tabular}{|c|c|}
\hline CHOP & Ciclos cada 21 días \\
\hline Ciclofosfamida & $750 \mathrm{mg} / \mathrm{m}^{2}$ iv día 1 \\
\hline Doxorrubicina & 50 mg/m² iv día 1 \\
\hline Vincristina & $1,4 \mathrm{mg} / \mathrm{m}^{2}$ iv día 1 \\
\hline Prednisona & $100 \mathrm{mg}$ vo días $1-5$ \\
\hline EPOCH & Ciclos cada 21 días \\
\hline Etopósido & $50 \mathrm{mg} / \mathrm{m}^{2}$ iv días $1-4$ \\
\hline Vincristina & 0,5 mg/día iv días 1-4 \\
\hline Ciclofosfamida & $750 \mathrm{mg} / \mathrm{m}^{2}$ iv día 4 \\
\hline Doxorrubicina & $10 \mathrm{mg} / \mathrm{m}^{2}$ iv días $1-4$ \\
\hline Prednisona & 100 mg/día vo días 1-5 \\
\hline Radioterapia & Al finalizar quimioterapia \\
\hline & Rayos X, 6 o 20 MV \\
\hline & campos comprometidos \\
\hline & $\begin{array}{l}39.6 \text { Gy dosis total } \\
1.8 \text { Gy dosis diaria }\end{array}$ \\
\hline
\end{tabular}

MV: megavoltios

Gy: Gray

El proceso de etapificación incluyó examen físico, exámenes de laboratorio, tomografía axial computada de tórax, abdomen y pelvis, biopsia de médula ósea bilateral y cintigrama con galio. El seguimiento se realizó cada 3 meses el primer año, cada 4 meses el segundo año, cada 6 meses el tercer y cuarto año, y después anual con examen clínico y hemograma.

La SG se midió desde el diagnóstico hasta la muerte del paciente o último control. La SLE desde el diagnóstico hasta la recaída tumoral, muerte o último control. Para las curvas de sobrevida se empleó el método de Kaplan y Meier. La comparación de las curvas de sobrevida fue realizada según el método de Log-rank. Se analizaron las toxicidades agudas de la quimioterapia y radioterapia según escalas de toxicidad de la OMS y RTOG (Grupo Oncológico de Radioterapia), respectivamente. Se realizó análisis multivariado por modelo de Cox incluyéndose las variables edad, sexo, presencia de síntomas $B$, esquema de quimioterapia empleado, número de ciclos utilizados y origen nodal/extranodal del linfoma. La comparación de variables dicotómicas se realizó mediante la prueba de $\mathrm{x}^{2} 0$ prueba de Fisher, con $\mathrm{p}$ significativo $<0,05$.

\section{RESULTADOS}

Se incluyeron 39 pacientes, 20 hombres y 19 mujeres, con el diagnóstico realizado entre agosto de 1996 y octubre de 2003. La edad media al diagnóstico fue de 57 años (20-85 años), 49\% (19 pacientes) fueron mayores de 60 años. El seguimiento medio fue de 51 meses (6-115 meses) (Tabla 2).

El 54\% de los pacientes presentaban etapa I de Ann Arbor. Cinco pacientes tenían síntomas B al diagnóstico. En relación al origen del linfoma, 17 pacientes (44\%) fueron diagnosticados en sitios extranodales, siendo la principal localización (24\%) el estómago. La mayoría de los pacientes (63\%) presentó al diagnóstico un IPI bajo (0-1). En relación a la quimioterapia utilizada $66 \%$ fue tratado con esquema CHOP y $34 \%$ con esquema EPOCH con un número de ciclos totales entre 3-6, dependiendo de la decisión del médico tratante. Al final del tratamiento todos los pacientes estaban en respuesta completa.

La histología más frecuente correspondió a linfomas difusos de células grandes (88\%). Otras histologías encontradas corresponden a linfomas difusos de células pequeñas, difusos de células pequeñas y grandes, foliculares de células grandes y del manto.

En relación a la toxicidad por quimioterapia, cerca de $90 \%$ de los pacientes presentaron toxicidad leve, principalmente por complicaciones neurológicas (neuropatía por vincristina), gastrointestinales (náuseas y vómitos) y mucositis. De 22 pacientes en quienes se obtuvo los registros clínicos, 58\% presentaron neutropenia grados 3-4 (neutrófilos totales menores a $1.000 \mathrm{x} \mathrm{ml}$ en el nadir). De 20 pacientes en quienes se pudo obtener la información, 43\% reportaron algún episodio de neutropenia febril de corta duración. No se reportaron muertes asociadas a quimioterapia.

Todos los pacientes recibieron radioterapia, independiente del número de ciclos o esquema de quimioterapia empleados. La toxicidad reportada para el tratamiento de radioterapia según la escala RTOG, en más de $90 \%$ de los pacientes estuvo ausente o fue leve, limitándose a los campos irradiados (radiodermitis, mucositis orofaríngea) y no dejó secuelas.

Al momento del análisis estadístico se han constatado 12 pacientes en primera recidiva (31\%) 
Tabla 2. Características clínicas de los pacientes tratados

\begin{tabular}{|ll|}
\hline Número de pacientes & 39 (20 hombres) \\
Seguimiento medio & 51 meses (6-115) \\
Etapa (Ann Arbor) & $54 \%$ etapa I (21 pacientes) \\
& $46 \%$ etapa II (18 pacientes) \\
Síntomas B & 4 pacientes $(10 \%)$ \\
Lugar primario & 17 extranodales $(44 \%)$ \\
& $24 \%$ estómago \\
Puntaje IPI & $63 \%$-1 \\
Tipo QT & $66 \%$ CHOP \\
No ciclos 3-4 & $34 \%$ EPOCH \\
5-6 & 14 pacientes $(36 \%)$ \\
Histologías & 27 pacientes $(64 \%)$ \\
Difuso células grandes & \\
Difuso células pequeñas & 34 pacientes $(88 \%)$ \\
Difuso células pequeñas y grandes & 1 paciente $(2 \%)$ \\
Folicular células grandes & 1 paciente $(2 \%)$ \\
Manto & 4 pacientes $(6 \%)$ \\
Recidiva (\%) & 1 paciente $(2 \%)$ \\
Fallecidos $(\%)$ & 12 pacientes $(31 \%)$ \\
\hline
\end{tabular}

y 8 pacientes fallecidos (20\%). La SG a 5 años fue de $72,4 \%$ (rango 53-88\%), y la SLE de 63,3\% (rango 40-78\%) (Figuras 1y 2).

Se realizó análisis comparativo de SG y SLE entre aquellos pacientes que recibieron 3-4 ciclos de quimioterapia y aquellos que recibieron 5 o más ciclos, no encontrándose diferencias estadísticamente significativas. La SG a 5 años de los pacientes que recibieron hasta 4 ciclos de QT y los que recibieron 5 y más fue de $52 \%$ y $78 \%$, respectivamente $(p=0,7$, log-rank). En tanto la SLE a 5 años fue de $75 \%$ y $59 \%$, respectivamente ( $p=0,54$, log-rank).

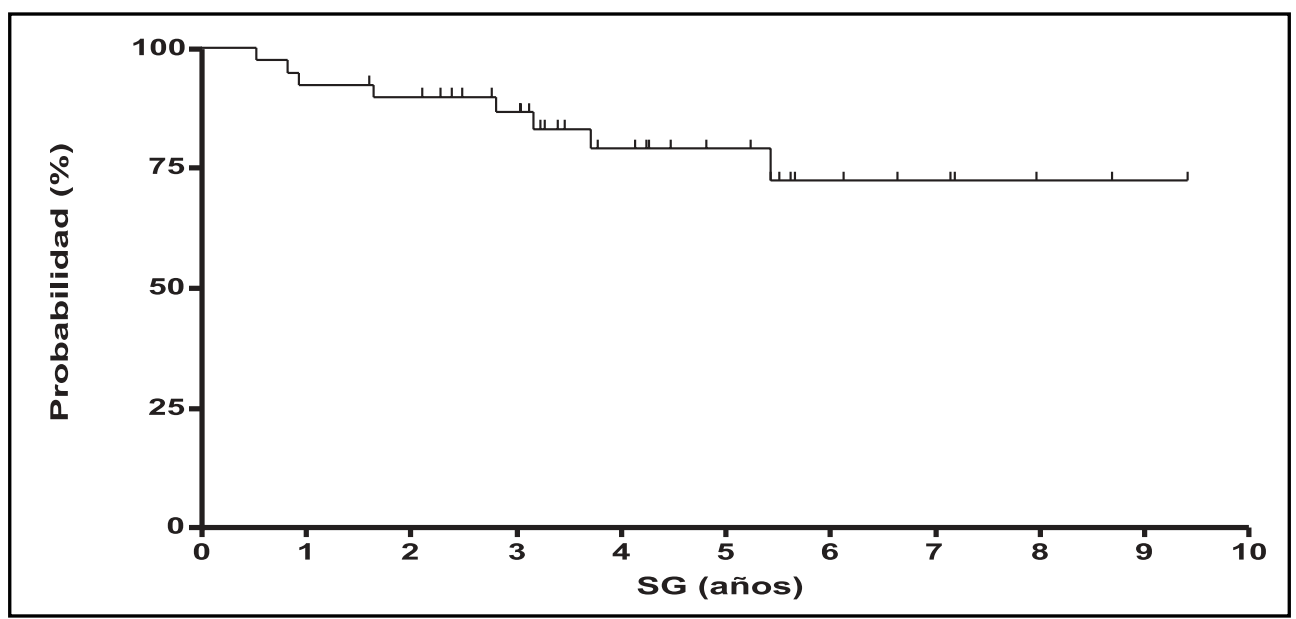

Figura 1. Sobrevida global (SG) linfoma no Hodgkin localizado etapas I y II. 


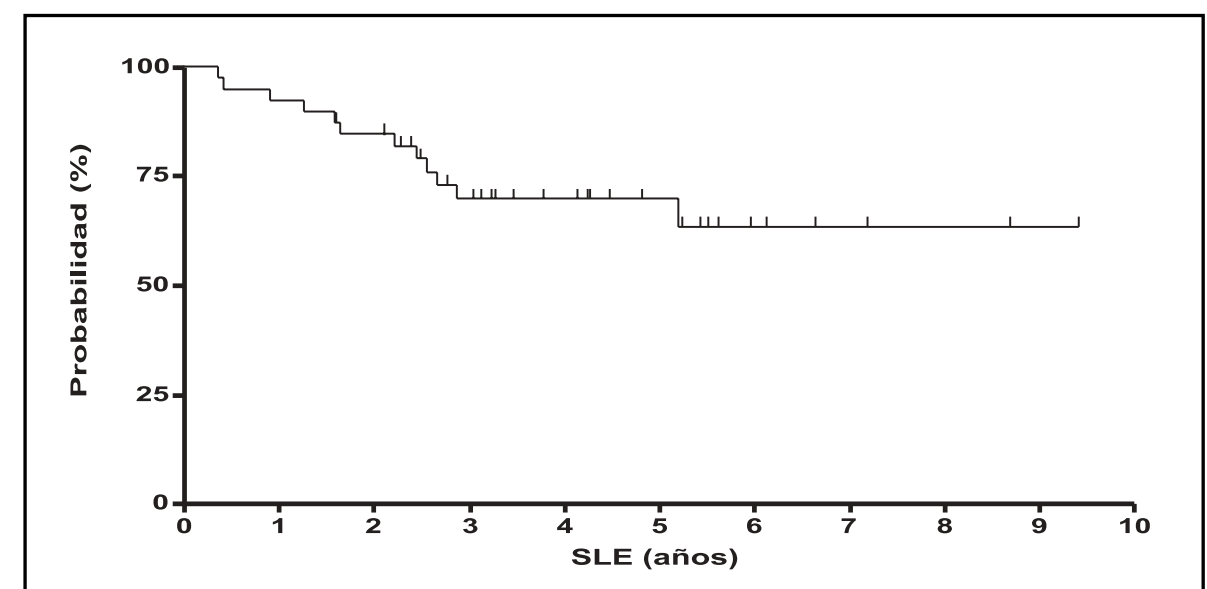

Figura 2. Sobrevida libre de eventos (SLE) linfoma no Hodgkin localizado etapas I y II.

El análisis de sobrevida según esquema de quimioterapia empleado, $\mathrm{CHOP}$ o $\mathrm{EPOCH}$, no demostró diferencias significativas en SG o SLE. La única variable asociada a SG y SLE correspondió a la edad de diagnóstico; la SG a 5 años de los grupos $<60$ y $>60$ años fue de $100 \%$ y de $49 \%$, respectivamente ( $p=0,002$, log-rank). La SLE a 5 años fue de $76 \%$ y de $52 \%$, para los grupos $<60$ y $>60$ años, respectivamente ( $p=0,03$, log-rank) (Figuras 3 y 4). El análisis multivariado por modelo de Cox, sólo mostró influencia de la edad en la SG, no así en la SLE.

\section{Discusión}

En las últimas dos décadas el objetivo de los tratamientos del LNH localizado, de grados intermedio y alto, ha sido el buscar terapias específicas dirigidas al tumor disminuyendo los efectos secundarios a corto y largo plazo ${ }^{1}$. La mayoría, $75 \%$, de los LNH de células B se diagnostican en etapas avanzadas y la base del tratamiento es la poliquimioterapia. Sólo en los últimos años la adición de anticuerpos monoclonales contra la molécula CD20 sobreexpresada en la superficie de células

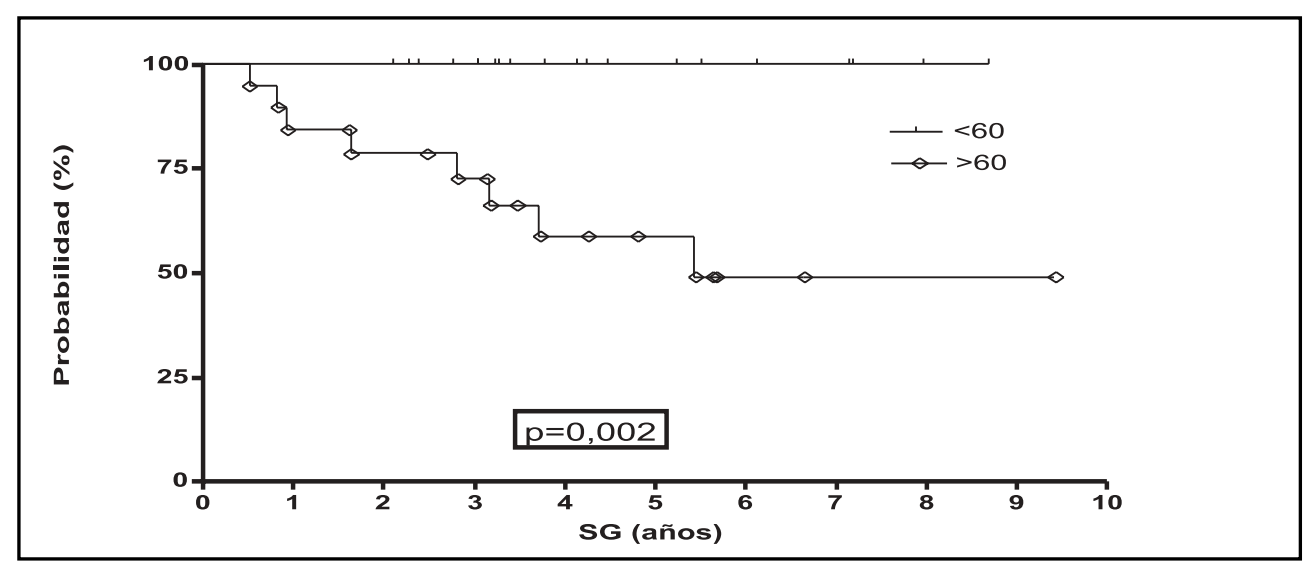

Figura 3. Sobrevida global (SG) según grupo etario ( $<60$ y $>60$ años) y comparación por Log-rank. Pacientes portadores de linfoma no Hodgkin localizado, etapas I y II. 


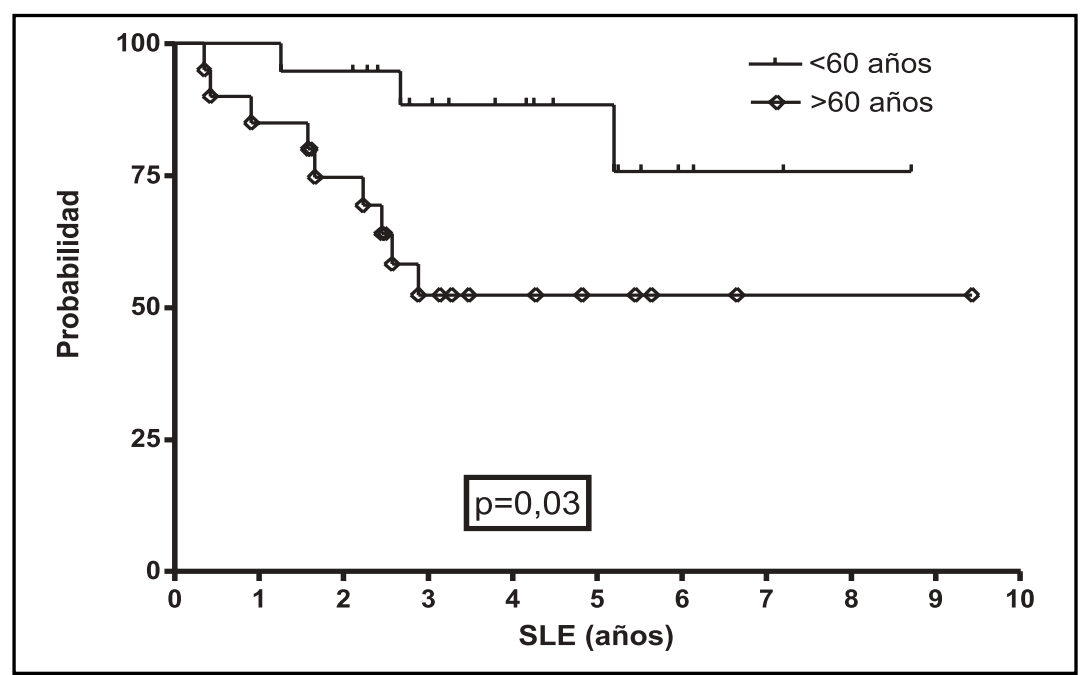

Figura 4. Sobrevida libre de eventos (SLE) según grupo etario ( $<60$ y >60 años) y comparación por Log-rank. Pacientes portadores de linfoma no Hodgkin localizado, etapas I y II.

tumorales ha demostrado beneficios en la sobrevida de estos pacientes comparada con poliquimioterapia exclusiva ${ }^{14}$.

En el LNH la recurrencia ocurre entre los 24-36 meses en la mayoría de los casos. El seguimiento medio de nuestra serie fue de 48 meses, cubriendo adecuadamente este periodo.

En relación con las características de nuestros pacientes, la edad de diagnóstico es similar a lo comunicado en las series de Miller y Shenkier y levemente mayor en la reciente serie publicada por Reyes, con una edad media de 46 años ${ }^{4,11,15}$. La proporción de pacientes sobre 60 años (46\%) también es semejante a lo comunicado por Miller (50\%) para el grupo tratado con quimioterapia y radioterapia.

En el presente trabajo, la proporción de pacientes con presentación nodal y extranodal fue similar a lo descrito, pero diferente en cuanto al estadio, ya que nuestra serie tiene una mayor cantidad de pacientes en etapa II. En series internacionales predominan las etapas I. En nuestra serie se mantiene también el predominio de los LNH difusos de células grandes sobre otras histologías.

No hubo diferencias en los resultados al comparar los esquemas CHOP y EPOCH. La serie de Khaled tampoco reportó diferencias entre los 2 esquemas, al compararla en 38 pacientes portadores de LNH agresivos etapas I-IV ${ }^{16}$.

La combinación de quimioterapia y radioterapia fue bien tolerada y de baja toxicidad. Los episodios de neutropenia febril fueron de corta duración y sin mortalidad asociada. La frecuencia de episodios febriles en nuestra serie fue de $43 \%$, que es inferior a $54 \%$ reportado por Miller para un tratamiento similar. En nuestra serie el número de ciclos y esquemas de quimioterapia fue heterogéneo, por lo que es posible que el número de episodios de neutropenia febril pudiera ser menor si se utilizaran 4 ciclos de quimioterapia.

Nuestros resultados de SG y SLE son comparables con los de las grandes series prospectivas publicadas con similares tratamientos de radioterapia y quimioterapia. (Tabla 3). No detectamos diferencias al usar 4 o más ciclos de quimioterapia. A pesar de nuestro reducido número de casos, la probabilidad de sobrevida a 5 años es similar a lo publicado por otros grupos, lo que apoya la efectividad de la quimioterapia acortada asociada a radioterapia. El efecto negativo de la edad mayor de 60 años en la SG y SLE fue demostrado en nuestra serie al igual que en otras series reportadas.

Respecto del uso de Rituximab, en nuestra serie sólo hay tres pacientes tratados con este 


\section{Tabla 3. Comparación de resultados de sobrevida global y sobrevida libre de recurrencia entre distintos trabajos publicados}

\begin{tabular}{|lllll|}
\hline & Miller, 1998 & Shenkier, 2002 & Reyes, 2005 & Ramírez, 2006 \\
\hline $\begin{array}{l}\text { Sobrevida global, } \\
5 \text { años }\end{array}$ & $82 \%$ (77-87) & $80 \%$ & $81 \%(77-86)$ & $72 \%(54-90)$ \\
$\begin{array}{l}\text { Sobrevida libre eventos } \\
5 \text { años }\end{array}$ & $77 \%$ (72-83) & $81 \%$ & $74 \%(69-78)$ & $63 \%(45-81)$ \\
\hline
\end{tabular}

anticuerpo monoclonal. Un estudio fase 2 del grupo americano SWOG (Grupo Oncológico del Sudoeste) en LNH localizados agresivos, demostró beneficio en respuesta y mortalidad al comparar los 62 pacientes tratados con 4 ciclos de rituximab y 3 ciclos CHOP más radioterapia contra una serie histórica tratada con 3 ciclos CHOP más radioterapia ${ }^{17}$.

Como conclusión de nuestra serie retrospectiva, demostramos que los pacientes con LNH localizado de grados intermedio y alto tratados con quimioterapia, en base a doxorrubicina y radioterapia a campos comprometidos, tienen una SG y SLR similar a lo comunicado en series internacionales. No existe diferencia significativa entre los pacientes que recibieron quimioterapia

\section{REFERENCIAS}

1. Fisher R, Gaynor E, Dahlberg S, Oken M, Grogan T, Mize E ET AL. Comparison of a standard regimen (CHOP) with three intensive chemotherapy regimens for advanced non-Hodgkin's lymphoma. N Engl J Med 1993; 328: 1002-6.

2. The International Non-Hodgkin's Lymphoma Prognostic Factors Project. A predictive model for aggressive non-Hodgkin's lymphoma. The International Non-Hodgkin's Lymphoma Prognostic Factors Project. N Engl J Med 1993; 329: 987-94.

3. Fisher RI, Devita VT, Johnson BL, Simon R, Young R et AL. Prognostic factors for advanced diffuse histiocytic lymphoma following treatment with combination chemotherapy. Am J Med 1977; 63: 177-82.
CHOP versus EPOCH, asociado a radioterapia, como tampoco el hecho de utilizar 5 ó 6 ciclos respecto de utilizar 3 ó 4 ciclos. Por el momento no es posible evaluar el rol del rituximab en nuestros pacientes dado el escaso número tratado y el poco seguimiento de estos pacientes.

En la actualidad está siendo de gran importancia la incorporación de estudios moleculares del tumor y su relación con respuesta al tratamiento, toxicidad, SLE y SG ${ }^{18}$. Esto permitirá comprender mejor los mecanismos involucrados en la génesis y desarrollo de los linfomas, para planificar terapias ajustadas a la agresividad de cada tumor, a las características de cada paciente, y en último término nos permitirá planificar estrategias de prevención en pacientes de alto riesgo.

4. Reyes F, Lepage E, Ganem G, Molna T, Brice P, COIFFIER B ET AL. ACVBP versus CHOP plus radiotherapy for localized aggressive lymphoma. N Engl J Med 2005; 352: 1197-205.

5. Kaminski M, Coleman N, Colby T, Cox R, Rosenberg S et AL Factors predicting survival in adults with stage I and II large-cell lymphoma treated with primary radiation therapy. Ann Intern Med 1986; 104: 747-56.

6. Hoppe R. The role of radiation therapy in the management of the non-Hodgkin's lymphomas. Cancer 1985; 55(9 Suppl): 2176-83.

7. Jones S, Grozea P, Metz E, Haut A, Stephens R, MORRISON F ET AL. Superiority of adriamycincontaining combination chemotherapy in the treatment of diffuse lymphoma: a Southwest Oncology Group study. Cancer 1979; 43: 417-25. 
8. Cabanilas F, Bodey G, Freireich E. Management with chemotherapy only of stage I and II malignant lymphoma of aggressive histologic types. Cancer 1980; 46: 2356-9.

9. Lester J, Fuler L, Conrad F, Sumvan J, Velásquez W, ButLeR J ET AL. The roles of staging laparotomy, chemotherapy, and radiotherapy in the management of localized diffuse lange cell lymphoma: a study of 75 patients. Cancer 1982; 49: 1746-53.

10. Connors J, Kumo P, Fairey R, Voss N et al. Brief chemotherapy and involved field radiation therapy for limited-stage, histologically aggressive lymphoma. Ann Intern Med 1987; 107: 25-30.

11. Miuer T, Dahlberg S, Cassady R, Adelstein D, Spier C, Grogan T ET AL. Chemotherapy alone compared with chemotherapy plus radiotherapy for localized intermediate- and high-grade non-Hodgkin's lymphoma. N Engl J Med 1998; 339: 21-6.

12. Carbone P, Kaplan H, Musshoff K, Smithers D, Tubiana M. Report of the Committee on Hodgkin's Disease Staging Classification. Cancer Res 1971; 31: 1860-1.

13. JANCAR J. Progress in the classification of hematological neoplasms. From REAL to WHO concept. Adv Clin Path 2000; 4: 87-97.

14. Coiffier B, Lepage E, Briere J, Herbrecht J, Tily H, BouabDalah R ET aL. CHOP chemotherapy plus rituximab compared with $\mathrm{CHOP}$ alone in elderly patients with diffuse large-B-cell lymphoma. $\mathrm{N}$ Engl J Med 2002; 346: 235-42.

15. Shenkier T, Voss N, Fairey R, Gascoyne R, Hoskins P, KLASA R ET AL. Brief chemotherapy and involvedregion irradiation for limited-stage diffuse largecell lymphoma: an 18-year experience from the British Columbia Cancer Agency. J Clin Oncol 2002; 20: 197-204.

16. Khaled H, Zekri Z, Mokhtar N, Ali N, Darwish T, ElatTAR I ET aL. A randomized EPOCH vs CHOP front-line therapy for aggressive non-Hodgkin's lymphoma patients: long-term results. Ann Oncol 1999; 10: 1489-92.

17. Milier T, Unger J, Spier C, Stea B, Cantu E, Leblanc $M$ ET AL. Effect of Adding Rituximab to Three Cycles of CHOP Plus Involved-Field Radiotherapy for Limited-Stage Aggresive Diffuse B-Cell Lymphoma (SWOG-0014). Abstracts of the American Society of Hematology 46th Annual Meeting. December 4-7, 2004, San Diego, California, USA. Blood 2004; 104 (11 Pt 2): 1b-416b.

18. Armitage J. Defining the stages of aggressive nonHodgkin's lymphoma-a work in progress. N Engl J Med 2005; 352: 1250-2. 